\title{
Laser Physics: Recent Clinical Trends
}

\author{
${ }^{1}$ Amit Dahiya, ${ }^{2}$ Minakshi Rana, ${ }^{3}$ Anita Hooda, ${ }^{4}$ Taruna Arora, ${ }^{5}$ Arun Kumar
}

\begin{abstract}
Dental lasers are becoming an integral part of contemporary dentistry. Lasers can artistically sculpt soft tissues while providing precision, increased visibility, improved impression techniques; predictable healing with decreased scarring, reduced anesthesia and postoperative swelling. With the introduction of the hard-tissue laser, cosmetic bonding procedures are more predictable by providing enhanced bond strength, more comfortable by requiring little to no anesthesia, and less invasive via controlled removal of tooth structure. To protect the faith of patients on the clinician, we must be familiar with the fundamentals of laser physics and tissue interaction so that the proper laser device is used to obtain the treatment objective safely and effectively. This review will discuss about laser physics in detail to make its use more scientific and reliable for both clinicians and patients.
\end{abstract}

Keywords: Laser physics, Physical properties, Laser, Interaction, Tissue effects.

How to cite this article: Dahiya A, Rana M, Hooda A, Arora T, Kumar A. Laser Physics: Recent Clinical Trends. Int J Laser Dent 2014;4(3):74-78.

\section{Source of support: Nil}

Conflict of interest: None

\section{INTRODUCTION}

The advancement in science and technology in the medical field amends a path for embedding new treatment modalities to the challenges presented by the viable diseases. The increasing use of lasers in dentistry and

\footnotetext{
${ }^{1}$ Senior Resident, ${ }^{2,4}$ Postgraduate Student

${ }^{3}$ Senior Professor, ${ }^{5}$ Assistant Professor

${ }^{1}$ Department of Orthodontics, Post Graduate Institute of Dental Sciences, Rohtak, Haryana, India

${ }^{2}$ Department of Periodontology, Manav Rachna Dental College, Faridabad, Haryana, India

${ }^{3}$ Department of Oral Anatomy, Post Graduate Institute of Dental Sciences, Rohtak, Haryana, India

${ }^{4}$ Department of Conservative Dentistry and Endodontics Government Dental College, Punjab, India

${ }^{5}$ Department of Pedodontics, Post Graduate Institute of Dental Sciences, Rohtak, Haryana, India

Corresponding Author: Amit Dahiya, Senior Resident Department of Orthodontics, Post Graduate Institute of Dental Sciences, Rohtak, Haryana, India, Phone: 9711550897 , e-mail: amitdahiya0909@gmail.com
}

medicine reflects the great advances in this technology during recent decades focusing more increasingly on using laser systems as an adjunct in periodontal therapy. In periodontics, the most commonly used are high-power lasers, such as $\mathrm{CO}_{2}$, Nd:YAG, and Er:YAG lasers, for calculus removal, osseous surgery, and soft-tissue management, such as gingivectomy, gingival curettage and melanin pigmentation removal, i.e. depigmentation. The physics behind the laser production and its interaction with the biological tissues is shuttered by its fascinating increased usage in dentistry. It is the duty of all of us to work in best interests of the patient than succumbing to the pressures from manufacturers to use technology when it is not warranted or indicated. It is ultimately the clinical judgment made by practitioner based on scientific evidence which makes all that differences in success of the treatment. Hence, a thorough knowledge regarding physics of laser production and its interaction with tissues will help us taking correct clinical judgment.

\section{HISTORY}

Light has been used as a therapeutic agent for many centuries. In ancient time, light was used in form of heliotherapy, which was later termed as phototherapy for various medical problems specially skin diseases. ${ }^{1,2}$ For example vitiligo, lupus vulgaris, etc. The word laser stands for 'light amplification by stimulated emission of radiation' (LASER). The first laser or 'MASER' was developed by Theodore H Maiman (1960). MASER is an acronym for 'microwave amplification by stimulated emission of radiation'. It was based on theory of spontaneous and stimulated emission of radiation given by Einstein, $1916 .{ }^{3}$ The first constructed LASER was a pulsed Ruby laser, which emitted light of $0.694 \mu \mathrm{m}$ wavelength. The main disadvantage of this laser as reported was damage to adjacent teeth and surrounding structures as a result of scattering of the laser beam. In 1968, Lobene at Forsyth Dental Center in Boston and Stern at UCLA shifted their attention to carbon dioxide laser, of larger wavelength $(10.6 \mu \mathrm{m})$ suitable for better absorption by enamel. This laser was considered for variety of hard tissue applications, such as pit and fissure sealants, welding of ceramic materials to enamel and prevention of dental caries. ${ }^{4}$ Melcher et al (1987) concluded that carbon dioxide laser could induce secondary dentin formation and sterilization of dentin and exposed pulp. ${ }^{5}$ Then, in 1974, Yamamoto 
from Tohoku University School of Dentistry in Japan introduced neodymium laser for its application to vital oral tissues. The thermal effects of radiation energy within the infrared range, such as Ruby and carbon dioxide, caused extensive damage to hard tissue and were equally injurious to dental pulp, which lead to search for better higher wavelengths like Er:YAG, holmium:YAG, Nd:YAG and excimer having potential to replace the dental drill for a number of uses. ${ }^{6}$

\section{LASER PHYSICS}

Lasers are unique and versatile instruments by virtue of their physical characteristics. Properties, such as collimation, coherence and monochromaticity, are generated in high energy processes occurring inside the optical cavity. ${ }^{7}$ To obtain the desired clinical effect of laser energy understanding laser fundamentals is must. The basic units or quanta of light are called as photons. These quanta are classified as cosmic rays, gamma rays, X-rays, light, microwaves or radio waves. The physical property that determines this classification of electromagnetic energy is wavelength. The wavelength is the distance a photon travels while electric field completes one oscillation. Laser light covers the range from about 0.1 to $10 \mu \mathrm{m}$ (Fig. 1). Short wavelength ultraviolet light is more energetic than long wavelength infrared. ${ }^{8}$ When

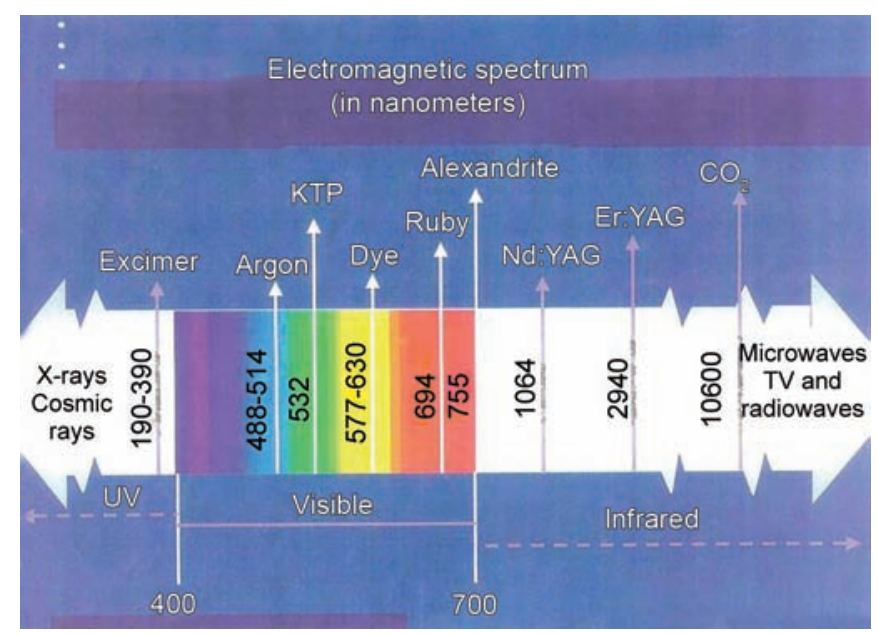

Fig. 1: Electromagnetic spectrum light encounters matter, it can be deflected (reflected or scattered) or absorbed. If a photon is absorbed, its energy is not destroyed but rather used to increase the energy level of absorbing atom and molecules. Such atoms will become unstable and will soon spontaneously decay back to ground state, releasing stored energy; this process is called as spontaneous emission (Figs 2A and B). The time interval between absorption and re-emission is very less and the photon released is also of less energy. This variation in energy is usually turned in to heat. Light amplification by stimulated emission of radiation is produced when an excited atom is stimulated to emit a photon before the process occurs spontaneously. ${ }^{9}$ This occurs by process of stimulated emission, where when a photon of right energy enters the electromagnetic field of an excited atom, the incident photon triggers the decay of excited electron to lower energy. This is accompanied by release of stored energy in form of second photon. The first photon is not absorbed but continues to encounter another excited atom. Stimulated emission can occur only when the incident photon has exactly the same energy as the released photon (Fig. 2C). ${ }^{10}$

\section{Laser Components and Beam Generation}

The basic components of laser include lasing medium placed in optical cavity, a pump energy source and a cooling system (Fig. 3). Optical cavity consists of two parallel mirrors placed on either side of the laser medium. One of the mirrors (M2) is completely reflective and another (M1) is transmissive. The light that escapes through M1 becomes laser beam, which is collimated, coherent and monochromatic. Mirrors bounce off the photons to the medium to stimulate the release of more photons. The mirror collimates the light, i.e. photons exactly perpendicular to mirrors re-enter the active medium, while those off-axis leave the lasing process. As the process is not $100 \%$ efficient, there is production of heat, so to counteract that cooling is provided via a coolant. Optical cavity contains active medium which has homogenous population of atoms or molecules that are pumped up to the excited state and stimulated to lase. ${ }^{11}$
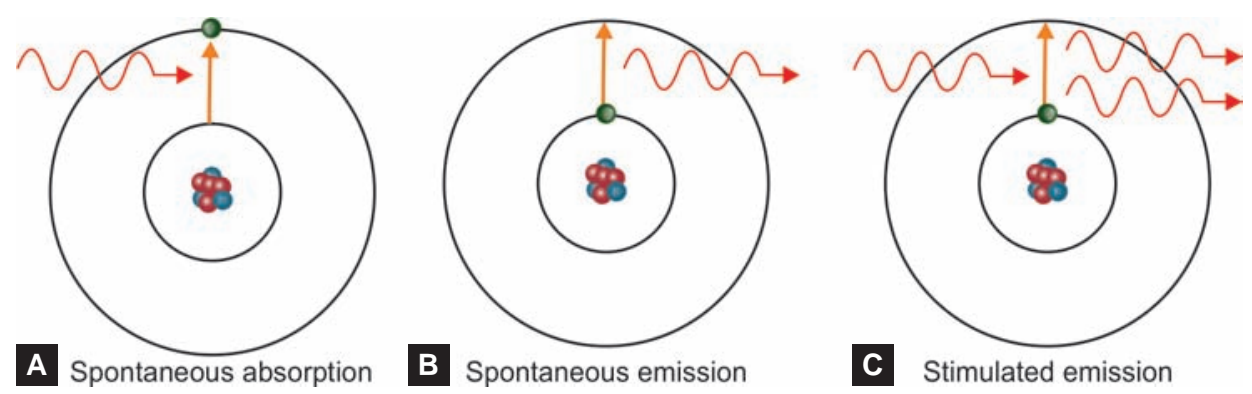

Figs 2A to C: Spontaneous absorption, emission and stimulated emission 


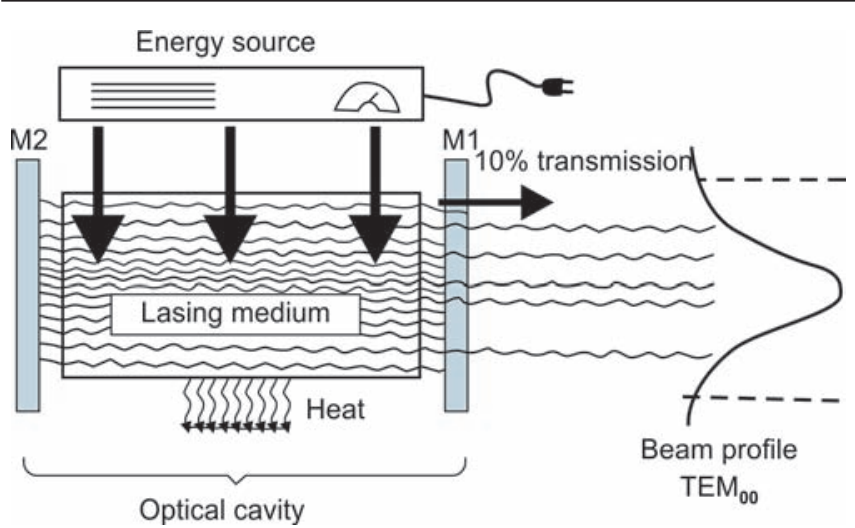

Fig. 3: Schematic diagram of a laser

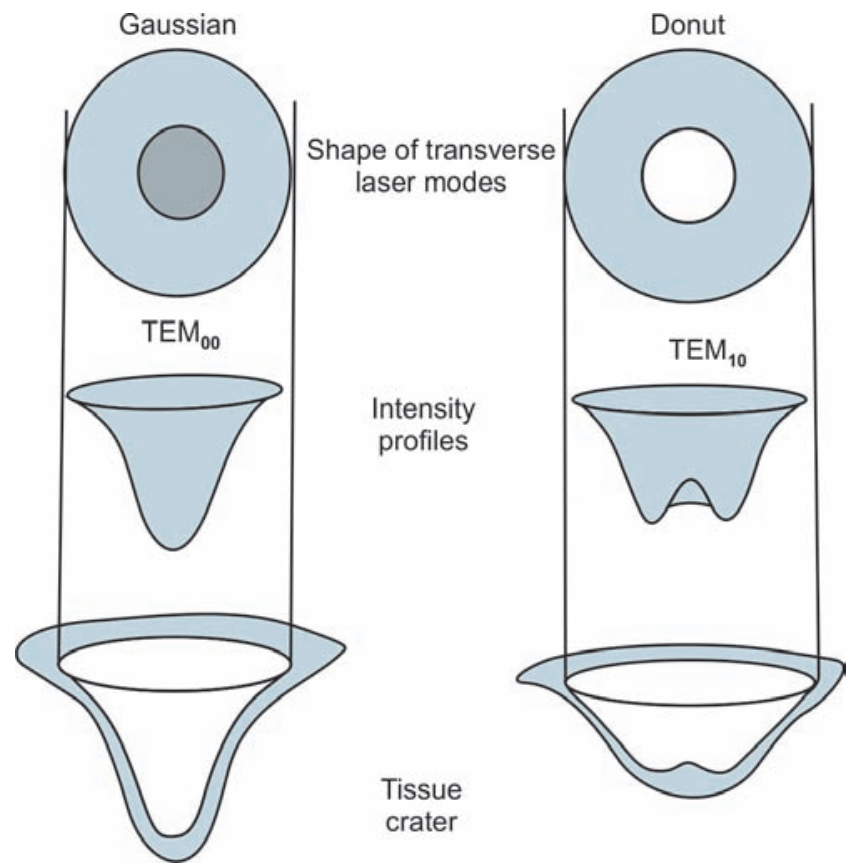

Fig. 4: Beam geometry

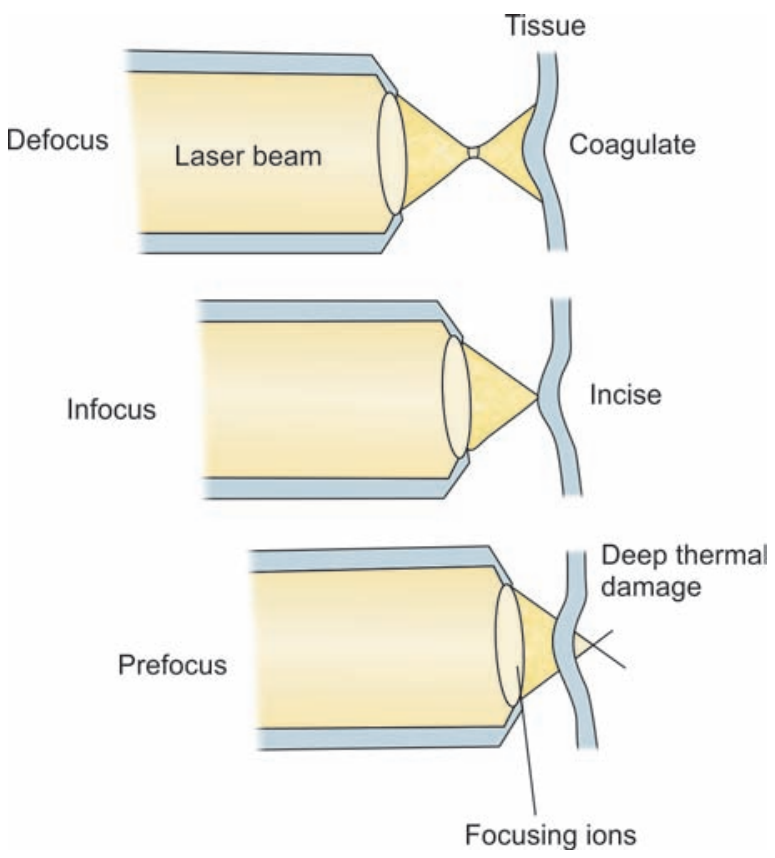

Fig. 5: Focusing modes of the laser beam

\section{PHYSICAL PROPERTIES OF LASER BEAM ${ }^{12}$}

- Beam profile: The cross section of beam is called as beam profile. There can be two beam profiles for an laser - fundamental or Gaussian beam profile and Donut profile (Fig. 4). In fundamental beam profile, the photons are concentrated more in the core of the beam and the light intensity diminishes at edges. Donut mode has a cold spot in center.

- Spot geometry: The projection of beam on target is called as 'spot'. The diameter of spot is called a spot size. Spot size got reduced with progressive increase in depth.

- Power density: It is the concentration of photon in unit area. Power density at center of spot is maximum and at edge it approaches to zero.

It is the play of power density and spot size of a laser that its interaction with tissues can be determined. Different power densities are achieved by combination of power and spot size. If spot size is reduced by 10 then power density increase by a factor of 100 . And if, power is increased by 10 then power density increases by 10 only. Hence, while using laser for incision purpose, the spot size is minimized so to improve the power density; whereas for coagulation purpose, the spot size is increased.

- Focal length and focal point: The lens determines the focal length. There are three terminologies used in this reference, i.e. focused, defocused mode and prefocused mode (Fig. 5). Laser should never be positioned with focal spot deep within tissues (prefocused) as this can lead to deeper thermal damage and undesirable tissue effect.

- Gating and pulsing: Surgical lasers have a mechanical shutter positioned in beam path. The opening and closing of circuit is controlled by a timing circuit. According to timing, they can be continuous, gated and pulsed (Fig. 6).

\section{Laser Interaction with Biological Tissues}

Essential elements determining interaction of laser with matter are wavelength, power density, temporal characteristic (pulsed or continuous), pulse rate and pulse duration. Other factors like mode of energy transfer attributable to delivery systems, such as contact or noncontact optical fiber delivery, and focused or unfocused beam. ${ }^{9}$ Certain biological factors also participate in determining tissue interaction more extensively like optical properties of various tissue elements, type of physiologic and mechanical processes that occur as a result of energy transformations within tissues. ${ }^{9}$ Out of these variables, the dependent variables include: specific wavelength of laser emission 


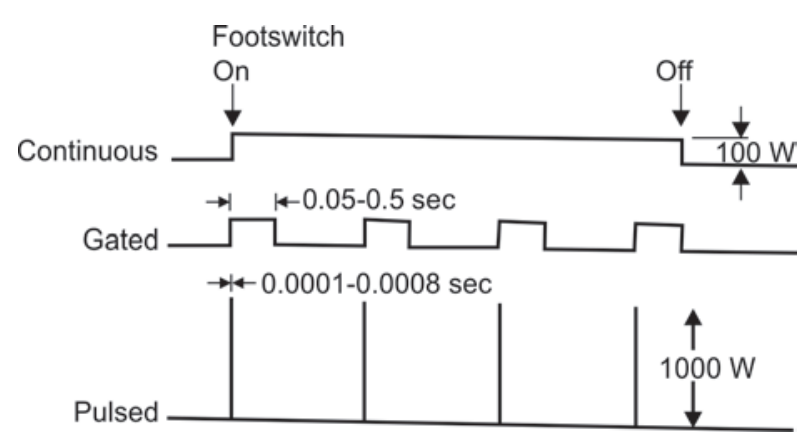

Fig. 6: Timing schemes for laser outputs

and optical characteristics of particular target tissue. Independent variables, which are under control of the clinician, include level of applied power (power density), total energy delivered (energy density), rate and duration of exposure and the mode of delivery (continuous or pulsed, contact or noncontact). Manipulation of all independent variables will enable operator to have precise control over the laser in the clinical setting to achieve desired tissue effect. ${ }^{13}$

\section{Dependent Variables (Optical Properties of Tissues and Wavelength Specificity)}

Four basic types of laser interaction with matter can be identified—reflection, absorption, transmission and scatter (Fig. 7). The extent of interaction will be proportional to the level of absorption of particular wavelength by tissue. Oral soft tissues are largely composed of water, which predominantly controls the tissue effects of laser emission within infrared spectrum, e.g. carbon dioxide laser. On the other hand, water is transparent to the emission of Nd:YAG laser, hence, accounting for its tendency to penetrate deeper into tissues. ${ }^{14}$ There are tissue elements that exhibit a high coefficient of absorption for particular wavelength called as chromophores. These chromophores have characteristic preferential absorption of specific wavelengths. Other chromophores within tissues, such as hemoglobin and melanin pigment, also exert a significant influence over interaction of radiant energy and tissues. Hemoglobin readily interacts with 488 and $514 \mathrm{~nm}$ wavelengths, thus accounting for greater ability of argon laser for coagulation and hemostasis. ${ }^{15}$

Oral hard tissues, such as enamel, dentin and bone due to variation in percentage composition of hydroxyapatite, show different optical behavior. Also, certain structural or morphologic interactions related to the direction of enamel rods and dentinal tubules are important. All the oral hard tissues show a favorable absorption, transmission and scattering properties in 2,000 $\mathrm{nm}$ region, which coincides with emission wavelength for thulium:YAG laser.

Preferential absorption by chromophores of particular laser wavelength provides for a phenomenon of

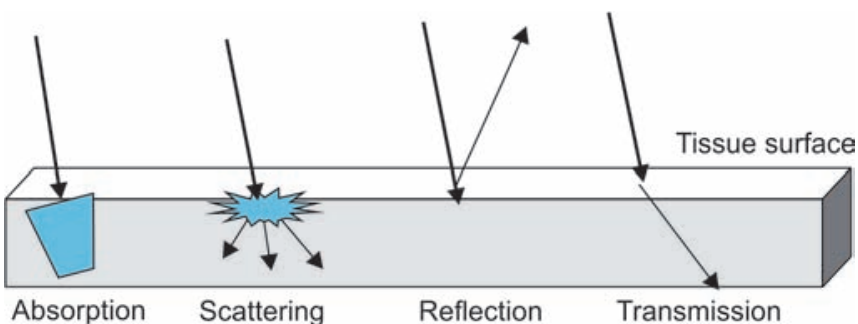

Fig. 7: Four basic types of laser interactions with matter

laser-tissue interaction known as wavelength or target specificity. ${ }^{15}$

\section{TISSUE EFFECTS OF LASER IRRADIATION}

When radiant energy is absorbed by tissue, four basic types of responses will be seen as follows:

- Photochemical interaction

- Photothermal interaction

- Photomechanical interaction

- Photoelectrical interaction

Photochemical interaction includes its clinical application in form of biostimulation, photodynamic therapy and tissue fluorescence. Basic principle behind the process is the interaction of specific wavelength with naturally occurring chromophores or dyes as photosensitizer to induce biologic reactions within tissue for both diagnostic and therapeutic applications. This interaction has been employed in the treatment of malignancies of the oral mucosa, particularly multifocal squamous cell carcinoma. It is also used in photo-polymerization of lightcured dental restorative materials.

Photothermal interaction manifest clinically as photoablation (removal of tissue by vaporization and superheating of tissue fluids), coagulation, hemostasis, photopyrolysis (burning away of tissue) and hepatocellular carcinoma.

Photomechanical interaction includes photodisruption or photodisassociation, which is breaking down of structures by laser. Photomechanical interaction is used for cavity preparation, caries and restorative materials removal.

Photoelectric interactions include photoplasmolysis, which is removal of tissue by formation by electrically charged ions. It has been recently used for nerve repair and regeneration.

The particular type of response produced by laser on interaction with matter is dependent on its wavelength, power density and exposure duration (Fig. 8).

\section{NEWER CONCEPTS OF LASER-TISSUE INTERACTION}

A laser beam interacts with matter by transferring its energy, which is converted into heat. For thermal 


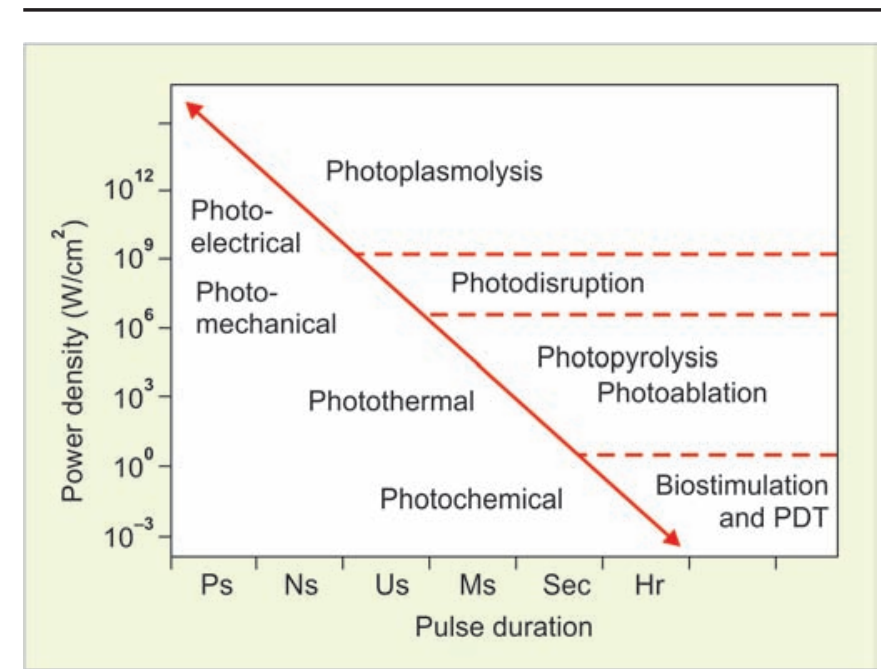

Fig. 8: Relationship of power density and interaction time with four lasers interaction with matter

modifications and matter evaporation to occur, the temperature of irradiated area has to pass a threshold to induce the substance phase change.

Continuous wave lasers are most commonly used for this process because they maintain a constant deposition of energy that produces a temperature high enough to induce the expected effect.

Thermal processes induced by laser beam interacting with matter are described in three stages as follows:

- Absorbed energy high enough to exceed internal energy to induce thermodynamic changes modify the lattice.

- Internal temperature increases to the level of melting point of matter and a change in phase occurs.

- Increasing temperature causes another phase change. Interaction between laser beam and third fluid medium can be induced to produce photomechanical ablation of tissue or other solid-state material. The laser interaction is initiated after structural modifications of the superficial layer of the material. The additional fluid is then involved in the interaction process but not until the fluid penetrates the microcracks and microporosities of modified layer. The photodisruption of matter occurs at relatively low levels of power density $\left(400-800 \mathrm{~W} / \mathrm{cm}^{2}\right)$.

\section{CONCLUSION}

A thorough understanding of complex process that occurs when specific wavelength of radiant energy interacts with biologic tissue is the prerequisite to the safe and effective application of lasers in the clinical practice. These complexities may be more easily derived once the inherent characteristics of different laser systems, modes of delivery and optical properties of oral soft and hard tissues are recognized. The effectiveness of dental laser therapy in clinical practice, therefore, depends on selection of the proper wavelength and use of appropriate parameters necessary to produce the desired tissue response for the particular clinical circumstances.

\section{REFERENCES}

1. Daniel MD, Hill JS. A history of photodynamic therapy. Aust NZ J Surg 1991;60(5):340-348.

2. American medical association. Council on scientific affairs. Lasers in Medicine and Surgery. JAMA 1986;256(4):900-910.

3. Acharya SS. Lasers in dentistry-a review. Annals and Essences of Dentistry 2012;IV(4):66-72.

4. Coluzzi D. Fundamentals of dental lasers: science and instruments. Dent Clin N Am 2004;48(4):751-770.

5. Melcher J, Chaumette F. Dental pulp exposed to the laser beam. Laser Surg Med 1987;7(4):347-352.

6. Yamamoto H. Ooya K. Potential of yttrium aluminium garnet laser in caries prevention. J Oral Pathol 1974;3(4):7-15.

7. Maiman TH. Stimulated optical radiation in Ruby. Nature 1960;187:493.

8. Castro DJ. The physical principles of laser. Atlas of Cutaneous Laser Surg 1992;1(1):235-256.

9. Dederich DN. Laser tissue interaction. Alpha Omegan 1991;84(4):33-36.

10. Dederick DN. Laser/tissue interaction: what happens to laser light when it strikes tissue? JADA 1993;124:57-61.

11. Hitz CB. Understanding laser technology Tulsa: Pennswell; 1991. p. 34-40.

12. Fuller TA, editor. Surgical laser: a clinical guide. Macmillan; 1987. p. 45-50.

13. Armon E, Laufer G. Analysis to determine the beam parameters which yield the most extensive cut with the least secondary damage. J Biomech Eng 1985;107:286-290.

14. Hardee M, Misrendeno JA. Evaluation of intracanal Nd:YAG laser irradiation (abstract). J Endodontics 1990;16:194.

15. Wright VC, Fisher JC. Laser surgery in gynecology. Philadelphia: WB Saunders; 1993;1:68. 